\title{
Pneumatic Actuator Controlled by Proportional Valve. Experimental results
}

\author{
Valentin Nicolae Cococi $^{1}$, Constantin Călinoiu ${ }^{1}$, and Carmen-Anca Safta $^{1 *}$ \\ ${ }^{1}$ University Politehnica of Bucharest, Power Engineering Faculty, 313 Splaiul Independentei, 060042, \\ Bucharest, Romania
}

\begin{abstract}
In nowadays the pneumatic controlled systems are widely used in industrial applications where valves must be operated, where there is a fire ignition risk, or in different automation systems where a positioning action is desired. The paper presents the experimental results of a pneumatic actuator controlled by a proportional control valve. The goal of the paper is to compare the experimental results with the numerical simulation results and to improve the mathematical model associated with the experiment.
\end{abstract}

\section{Introduction}

Electropneumatic control systems have the advantage that can be miniaturized and embedded in themselves electronic elements which allow them to be used in complex control systems [1]. So, new structures of pneumatic drives combined with electric components open the possibility for the use of programmable logic controller (PLC). This is an important advantage to use the electropneumatic systems in advanced control strategies which are characteristic to "Industry 4.0" philosophy [2].

This new perspective of electropneumatic devices used in so many industrial applications, medical systems, automotive structures, justify the theoretical and experimental approach of their study.

The aim of this work is to extend the theoretical studies of the pneumatic actuator controlled by proportional valve, in open loop and control loop, with experimental tests. In our previous studies [3-5] we considered the dynamic behaviour of a pneumatic actuator, controlled by a proportional valve, when different types of input signals where applied. Also, the electropneumatic servomechanism was loaded with variable technological force which can appear during the technological process or in an unexpected moment of time $[4,5]$.

In this paper, we present the results of the experimental study which is made on a pneumatic actuator controlled by a $5 / 3$ ways proportional valve. It was experimented if the actuator can follow the input signal applied with different frequencies and amplitudes to command the proportional valve. A static characteristic of the servo pneumatic system was plotted.

The experimental results were reconstructed by numerical simulation on a numerical model that have constructive parameters corresponding to the devices used in the

\footnotetext{
* Corresponding author: safta.carmenanca@gmail.com
} 
experimental stand. Are compared the result obtained in the experiments with the ones obtained in numerical simulations, and the conclusions are obtained to improve the experiments program.

The paper is organized in five sections. After a short introduction in which was underlined the aim of the paper, in the second section is presented the numerical simulation model of the pneumatic actuator and proportional valve that are included in the experimental test rig. Numerical simulations results are presented. In the third section is described the experimental bench and the test program. The experimental results and comments of them are the subject of the fourth section. In section five conclusions for further work are mentioned.

\section{Numerical simulation model and numerical results}

In the description of the numerical simulation model, we used the experience gained in the previous work [3-5] where it was considered a pneumatic actuator with the proportional pneumatic valve (PPV) in closed loop. The technical characteristics of the pneumatic actuator and PPV are the same with those used in the experimental setup.

It was used a proportional controller with proportional coefficient gain $K_{p}=1$, and an unloaded pneumatic actuator (the external force is zero).

In Figure 1 is given the numerical simulation scheme.

The pressure of the compressed air supplied in the system is 7 barA. The pneumatic actuator is a double-acting pneumatic cylinder with the length stroke of $450 \mathrm{~mm}$ and the piston active area $491,6 \mathrm{~mm}^{2}$. The mathematical model which describes the actuator behaviour consider the viscous friction coefficient of $75 \mathrm{Ns} / \mathrm{m}$ and thermal exchange coefficient of $50 \mathrm{~J} / \mathrm{m} 2 / \mathrm{K} / \mathrm{s}$ with an external temperature of $293.15 \mathrm{~K}$.

The PPV has the area of control orifices of $28,27 \mathrm{~mm}^{2}$ and the flow control coefficient of 0.72 . The valve limit frequency $(-3 \mathrm{~dB})$ is $100 \mathrm{~Hz}$ at 10 bar maximum pressure value and nominal voltage value at pneumatic mid-position of $5 \mathrm{~V}$ DC.

The liniar transducer used in the control scheme has a zero offset and the power supply of $10 \mathrm{~V} \mathrm{DC}$ with an effective electrical working distance of $457 \mathrm{~mm}$.

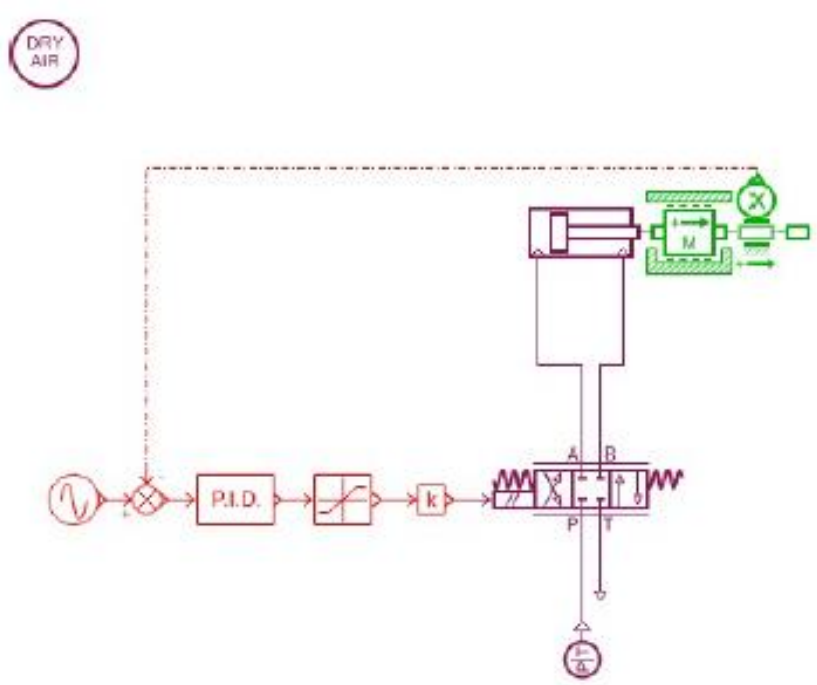

Fig.1. Numerical simulation model 
The reference input signal is plotted in Figure 2. The numerical results obtained for the input signal applied to the system are plotted in Figure 3.

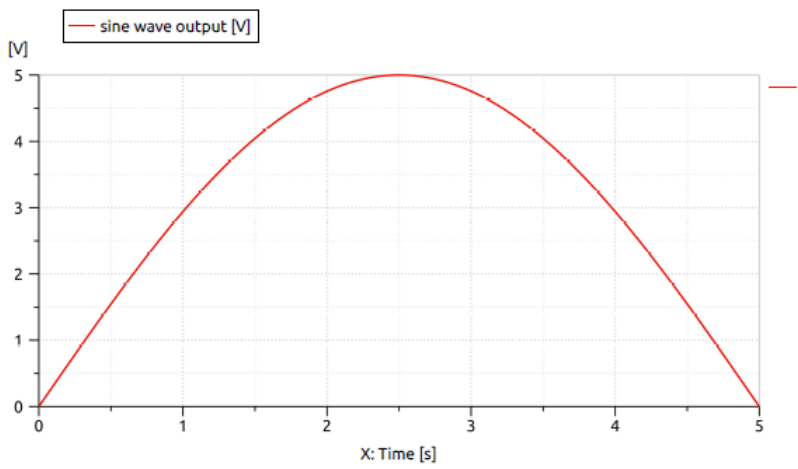

Fig 2. Time variation of the reference (input signal)

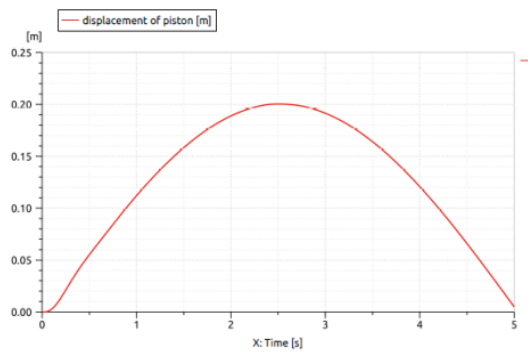

a)

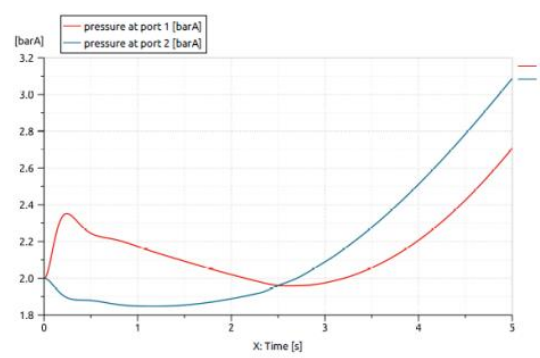

c)

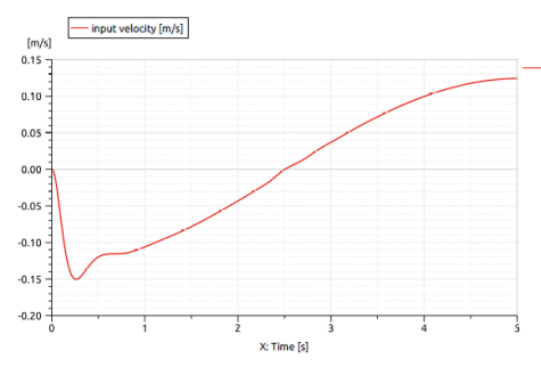

b)

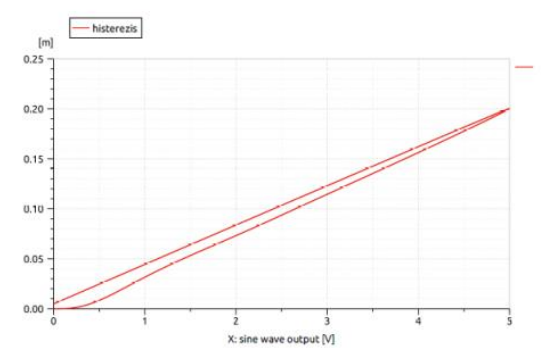

d)

Fig.3. Electropneumatic servomechanism dynamic and static behaviour: a) displacement of the piston; b) velocity of the piston; c) pressures in cylinder; d) static characteristic curve

It is observed that for an amplitude of $5 \mathrm{~V}$ of the input signal the maximum stroke obtained by the pneumatic actuator is $200 \mathrm{~mm}$ in 2.5 seconds.

In figure 3.b, the velocity pattern follows the displacement evolution and is observed the absence of external force. In the pressure distribution plot, "port 1" of the cylinder corresponds to the left chamber of the cylinder, Figure 1.

The hysteresis curve, Figure 3.d, is obtained by plotting the output signal versus input, the piston displacement. Even hysteresis curve underlines the absence of static friction within the system, caused by no external force. 


\section{Experimental setup}

For experiments we considered a pneumatic servo mechanism having a pneumatic actuator and a proportional pneumatic valve with the characteristics mentioned above. The experimental arrangement is show in Figure 4.a and the bloc diagram of the control system is presented in Figure 4.b.

The pneumatic servomechanism is supplied with air from the air compression unit (noted 1 in Figure 4.a). The proportional valve (noted 4) is controlled by the PID controller (noted 5) electrical supplied by 24 VDC power supply (noted 6). Also, the proportional valve will control the flow to the pneumatic actuator (noted 2) of which displacement is follow up by the displacement transducer (noted 3). The electrical signal from the transducer is compared with the reference input from the controller.

The Arduino board is used as a data acquisition system. The Arduino board is connected to the computer's USB port using a USB A-B cable, available in 1.5 meters or 3 meters. The Software used to read the data from the board is Arduino Integrated Development Environment (IDE) [6, 7].

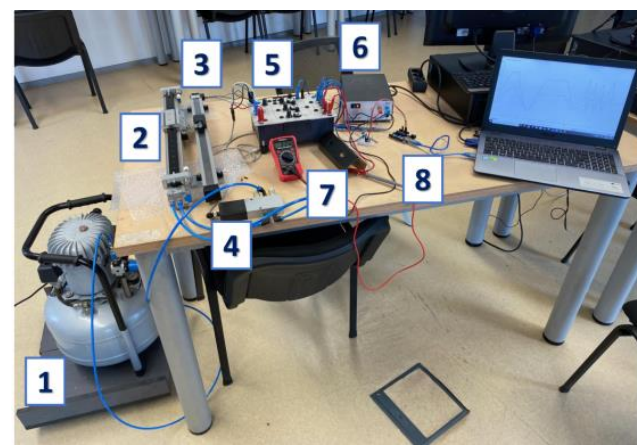

a)

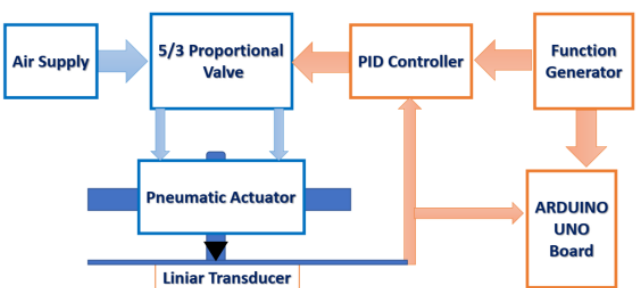

b)

Fig. 4. Experimental test stand: a) Position control components: 1-air supply, 2-pneumatic actuator, 3linear transducer, 4-proportional valve 5/3 ways, 5-PID controller, 6-24V power supply, 7multimeter, 8-Arduino UNO board; b) Block diagram

\section{Experimental results}

In the experiments were use input signals with different amplitudes and frequencies, as in Figure 5. The ability of the system to track the input signal was observed. The resolution of the data acquisition system is 10 bits, and the conversion range is $0 \ldots 5 \mathrm{~V}$.

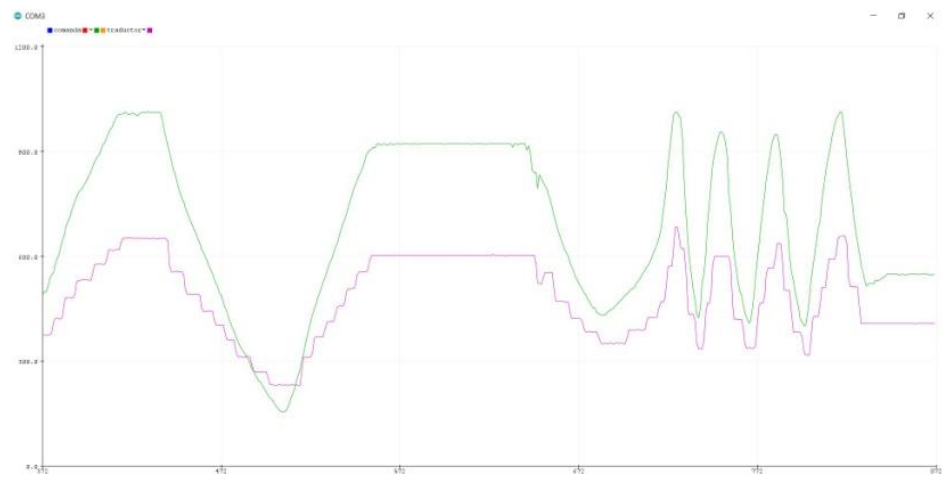

Fig. 5. Signals in bits: green line input signal, violet line-output signal 
In Figure 6 is plotted the experimental response of the servomechanism to the input signal. It is observed that the output signal follows the input, but it was found that the area of insensitivity/resolution of the drive system has values in the range of $100 \div 250 \mathrm{mV}$, depending on the position of the piston, with values higher towards the end of the stroke.

The relatively poor resolution $(10 \mathrm{~mm})$ of the drive system could be given by friction in the cylinder or by the performance of the servo controller. Another explanation of this result is given by the fact that the signal generator did not allow a control voltage of around $5 \mathrm{~V}$ which is the voltage corresponding to the neutral zone of the servomechanism. The experiments were performed with control signals below the value of $5 \mathrm{~V}$ and hence the difficulties in the experimental determinations.

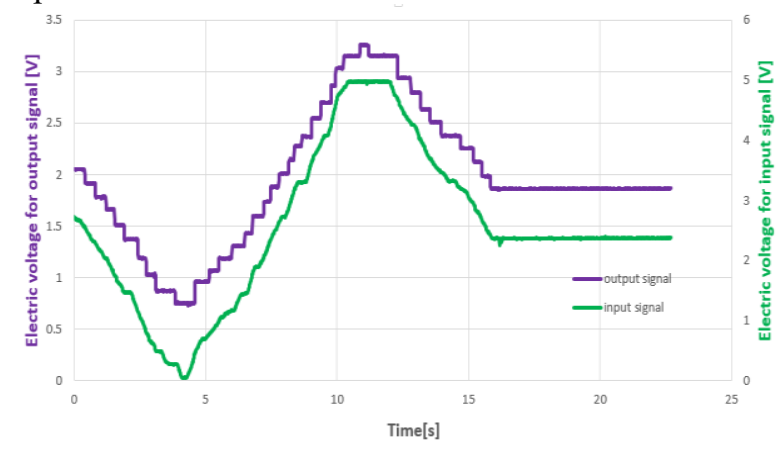

a)

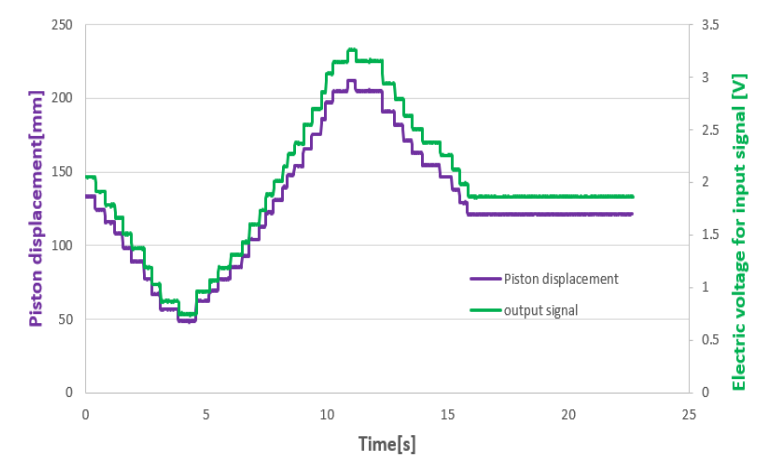

b)

Fig. 6. The variation of input signal and output signal: a) in electric voltage; b) in mm for the output signal

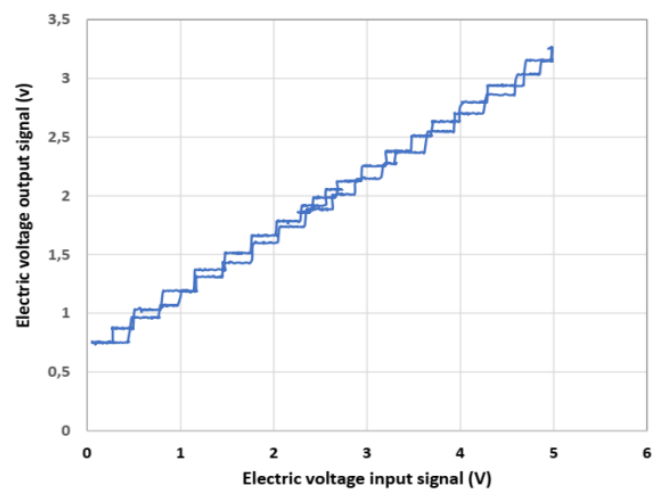

Fig. 7. Hysteresis curve 
In Figure 7 is plotted the static characteristic (or hysteresis curve) of the control system. It is observed that he static characteristic of the drive system is quasi-linear.

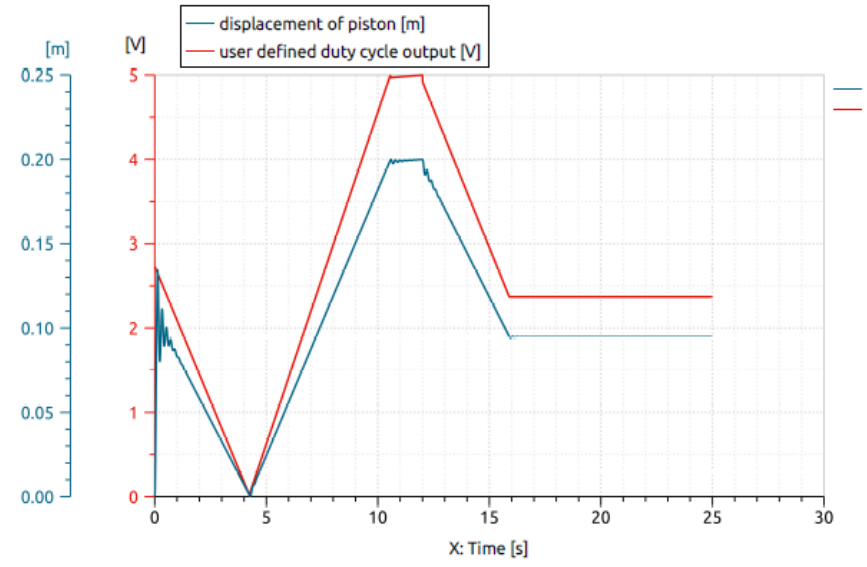

a)

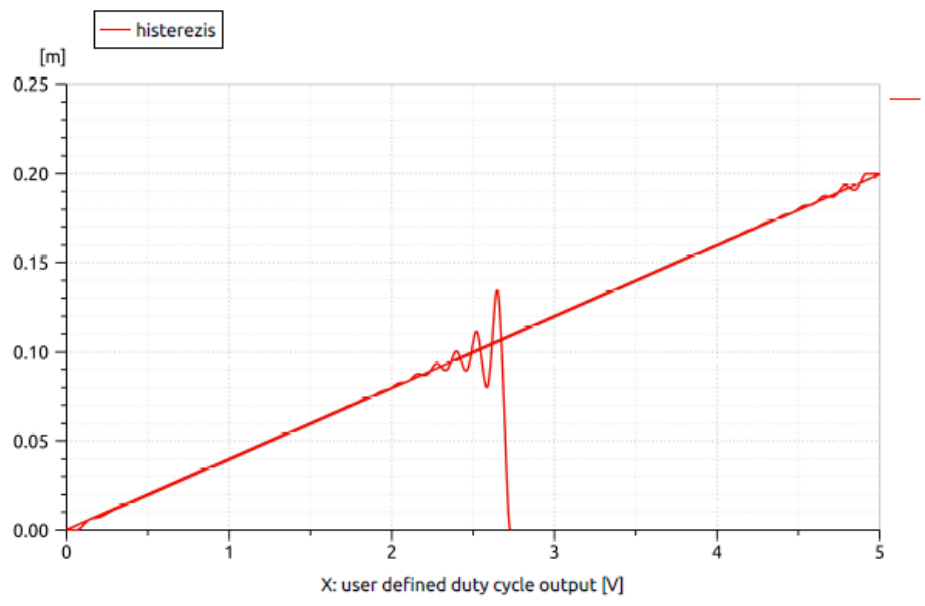

b)

Fig. 8. Simulation results for an input signal similar with the one in the experiments: a) evolution of the piston displacement; $b$ ) hysteresis curve

Finally, having the numerical model and the experimental results, we numerically build an input signal with an amplitude of $4.97 \mathrm{~V}$ as in Figure 6a which was applied in the numerical model. The response of the system is given in Figure 8. The numerical simulation results are comparable with the experiments (see Figure 6 and Figure 8.a). Even the quasi-linear characteristic of hysteresis curve is approximatively replicated. The drop observed in Figure $8 \mathrm{~b}$ is given by the sudden openings of the spool valve. How speed is a derivative of the displacement, on short period of time the values are big.

\section{Conclusions}

Based on the results of previous studies on the behavior of pneumatic positioning servomechanisms using numerical simulation models, experiments have been performed in this paper. 
Thus, the positioning system consisting of pneumatic actuator, proportional distributor, displacement transducer and controller was considered. For different input signals, the actuator's ability to track the input signal was observed. The hysteresis characteristic of the system was also found.

The obtained results showed that the positioning servomechanism can follow the input signal, but the resolution of the system is poor. This deficiency of the system may be caused by static friction, or by the characteristics of the controller.

Therefore, a more rigorous testing program is proposed to identify the causes of poor system resolution. It is also necessary to use a more efficient signal generator and to improve the test procedure regarding the dynamic behavior of pneumatic servomechanism.

We would like to thank the company FESTO Didactic Romania for lent us a part of the pneumatic equipment necessary to carry out the experiments.

\section{References}

1. W. Kobayashi, N. Kato, S. Dohta, T. Akagi, Position Control of Flexible Pneumatic Cylinder Using Tiny Embedded Controller with Disturbance Observer, Int. J. of Mechanical Engineering and Robotics Research Vol. 6, No. 4, 318-321 (2017)

2. K Foit, W Banaś, G Ćwikła, IOP Conf. Ser.: Mater. Sci. Eng. 400022024 (2018)

3. V.N. Cococi, C.-A. Safta, C. Calinoiu, Dynamic behaviour of pneumatic actuators in open-loop controlled by proportional valves, E3S Web of Conferences, 2020, 180, 04012

4. V.N. Cococi, C.-A. Safta, C. Calinoiu, Numerical simulation approach of a pneumatic actuator to force perturbation, EPE 2020 - Proceedings of the 2020 11th International Conference and Exposition on Electrical And Power Engineering, 2020, pp. 589-593, (2020) 9305558

5. V.N. Cococi, C.-A. Safta, C. Calinoiu, Parameter tuning process for a closed-loop pneumatic actuator, The 7th Conference of the Sustainable Solutions for Energy and Environment (EENVIRO 2020), (to be published)

6. F. Mohamed, A. A. Dahoud, Integrated Development Environment "IDE" For Arduino (2018) https://www.researchgate.net/publication/328615543 Integrated Development Enviro nment IDE For Arduino

7. https://docs.rs-online.com/406c/0900766b8133324b.pdf 